\title{
Low testosterone levels, depressive symptoms, and falls in older men: a cross-sectional study.
}

\section{$\operatorname{AUTHOR}(S)$ :}

Kurita, Noriaki; Horie, Shigeo; Yamazaki, Shin; Otoshi, Kenichi; Otani, Koji; Sekiguchi, Miho; Onishi, Yoshihiro; ... Konno, Shin-ichi; Kikuchi, Shin-ichi; Fukuhara, Shunichi

\section{CITATION:}

Kurita, Noriaki ...[et al]. Low testosterone levels, depressive symptoms, and falls in older men: a cross-sectional study.. Journal of the American Medical Directors Association 2014, 15(1): 30-35

\section{ISSUE DATE:}

2014-01

URL:

http://hdl.handle.net/2433/184352

\section{RIGHT:}

(C) 2014 Published by Elsevier Inc.; This is not the published version.

Please cite only the published version.; この論文は出版社版でありませ ん。引用の際には出版社版をご確認ご利用ください。 


\section{Low testosterone levels, depressive symptoms, and falls in older men: a cross-sectional study}

Noriaki Kurita, MD, FJSIM ${ }^{*}$; Shigeo Horie, MD, PhD ${ }^{2,3^{*}}$; Shin Yamazaki, PhD ${ }^{l}$; Kenichi Otoshi, MD, PhD ; Koji Otani, MD, PhD" ; Miho Sekiguchi, MD, PhD $;$; Yoshihiro Onishi, MPH, PhD ; Misa Takegami, RN, PhD ; Rei Ono, MPH, PhD"; Shin-ichi Konno, MD, PhD ; Shin-ichi Kikuchi, MD, PhD ; and Shunichi Fukuhara, $M D, D M S c^{1,8}$

Department of Healthcare Epidemiology, Graduate School of Medicine and Public Health, Kyoto University, Kyoto, Japan ${ }^{1}$

Department of Urology, Juntendo University School of Medicine, Tokyo, Japan²

Department of Urology, Teikyo University School of Medicine, Tokyo, Japan ${ }^{3}$

Department of Orthopedic Surgery, Fukushima Medical University School of Medicine, Fukushima, Japan ${ }^{4}$ Institute for Health Outcomes \& Process Evaluation Research (i-Hope international), Kyoto, Japan ${ }^{5}$

Department of Preventive Medicine and Epidemiologic Informatics, National Cerebral and Cardiovascular Center, Osaka, Japan ${ }^{6}$

Department of Community Health Sciences, Kobe University Graduate School of Health Sciences, Kobe, $\operatorname{Japan}^{7}$

Center for Innovative Research in Clinical Evaluative Science (CiRCLE), Fukushima Medical University, Fukushima, $\operatorname{Japan}^{8}$

*N.K. and S.H. contributed equally to this work.

Running title: testosterone and falls

Keywords: testosterone; depression; fall

Address correspondence to: Shunichi Fukuhara, MD, FACP

Department of Healthcare Epidemiology

Graduate School of Medicine and Public Health

Kyoto University 


\section{Yoshida Konoe-cho, Sakyo-ku}

Kyoto 606-8501, Japan

TEL: 81-75-753-4646

FAX: 81-75-753-4644

E-mail: fukuhara.shunichi.6m@kyoto-u.ac.jp 


\section{Abstract}

Objectives: While several studies have cited a potential association between testosterone deficiency and risk of falls among community-dwelling older men, evidence for such an association is conflicting. Depressive symptoms, which occasionally accompany testosterone deficiency but which are often neglected as associated symptoms, may actually provoke falls independent of or jointly with testosterone deficiency. We examined the association between testosterone levels, depressive symptoms, and falls and assess the joint effect of testosterone levels and depressive symptoms on falls among older men.

Design, Setting, and Participants: Data for this cross-sectional study were obtained from 869 men aged over 60 years from 2 Japanese municipalities who participated in health check-ups conducted in 2010. Salivary testosterone (sT) levels were measured using an enzyme-linked immunosorbent assay, and depressive symptoms were assessed via the short form of the Center for Epidemiologic Studies Depression Scale.

Main outcome measures: Self-reported "any fall" over the one-month period.

Results: Among the total of 482 participants analyzed (median age: 70 years), 10.8\% reported any fall. On comparison between 90-percentile sT levels and lower levels, our logistic regression model with restricted cubic splines showed that lower sT levels were associated with an increased likelihood of suffering any fall after adjustment for sociodemographic characteristics, comorbidities, and mobility function. For example, 5-percentile sT was associated with any fall (adjusted odds ratio $[\mathrm{OR}], 4.23[95 \%$ confidence interval $\{\mathrm{CI}\}, 1.66-10.8])$. Depressive symptoms were also strongly associated with any fall (adjusted OR, 3.49 [95\% CI, 1.52-8.04]). We noted no apparent interaction of sT and depressive symptoms with falls $(\mathrm{P}=0.079)$, suggesting that the joint effect of testosterone deficiency and depressive symptoms on falls was multiplicative. Indeed, compared with a combination of 90-percentile sT values and no depressive symptoms, adjusted OR for any fall in a combination involving 5-percentile sT and depressive symptoms was 14.8-fold (95\% CI, 3.76-58.0).

Conclusion: Our findings indicated that both relatively low testosterone levels and presence of depressive symptoms were independently associated with falls among older men. Causality of these associations should be confirmed in future prospective studies.

\section{Introduction}

Falls are a serious public health problem, with one third of community-dwelling individuals aged over 65 years falling at least once annually ${ }^{1}$ and with $5 \%$ to $10 \%$ of falls resulting in fracture, head injury, or other serious injuries. ${ }^{2}$ While falls in older individuals are multifactorial, age-related decline in lower extremity strength or mobility function are recognized as important contributors. ${ }^{3-5}$ Separately, age-related decline in testosterone levels in men is a growing concern, with approximately $30 \%$ of men aged 40-79 years showing testosterone deficiency. ${ }^{6}$ However, while previous studies have suggested that low testosterone levels are associated with reduced extremity strength or mobility function in older men, ${ }^{7,8}$ relatively few have examined the association between low testosterone levels and falls, with conflicting results despite the 
ostensibly plausible biological connections.

One concern with these previous studies examining the relationship between testosterone deficiency and falls is their lack of consideration for presence of depressive symptoms. ${ }^{9-11}$ Testosterone-deficient individuals are likely to have concurrent depressive symptoms, ${ }^{12}$ and depressive symptoms are also associated with falls. ${ }^{4}$ One study found no association between testosterone levels and incident falls after adjustment for confounders including depressive symptoms, ${ }^{13}$ suggesting the potential importance of examining the effects of testosterone deficiency on falls independent of depressive symptoms. In addition, if both testosterone deficiency and depressive symptoms are proven to be associated with falls, whether or not men with both testosterone deficiency and depressive symptoms are more likely to suffer falls than those with either condition alone would also be of clinical relevance, as these individuals are likely to be seen by physicians specialized in treating testosterone deficiency. ${ }^{14,15}$

Here, to examine whether or not testosterone deficiency and depressive symptoms are independently associated with falls in community-dwelling older men, we analyzed data from the Locomotive Syndrome and Health Outcome in Aizu Cohort Study (LOHAS). We further evaluated how the strength of the association between the composite of testosterone deficiency and depressive symptoms and falls is larger or smaller than the product of the strength of the association between testosterone deficiency and fall and that between depressive symptoms and falls.

\section{Method}

Our cross-sectional study was approved by the Research Ethics Committee of Fukushima Medical University School of Medicine. The LOHAS is a population-based study conducted starting in 2008 involving residents aged 40-80 years who participated in annual health check-ups among two communities (Tadami and Minamiaizu Towns) in Fukushima Prefecture, Japan. Eligibility criteria for this study were "aged over 60 years" and "participated in the health check-up conducted in 2010". No exclusion criteria were set. All participants provided written informed consent. Details of the design of the LOHAS have been reported previously. ${ }^{16}$

\section{Measurement of salivary testosterone}

Salivary concentration of testosterone (sT) was the main exposure, measured using an enzyme-linked immunosorbent assay (ELISA) on the RE52631 system (IBL International GmbH, Hamburg, Germany) and expressed in $\mathrm{pg} / \mathrm{mL}$ (to convert to picomoles per liter, multiply by 3.47). sT reflects the level of free testosterone in plasma. Saliva was collected in the morning, at least 30 minutes after breakfast. Participants cleaned their mouth out with water gently and were asked to avoid brushing their teeth. All subjects collected whole saliva by directly spitting into polypropylene tubes through a polypropylene straw-tube. If a subject had little saliva, he was instructed to chew gum prepared specially for saliva collection. The supernatants of saliva obtained after centrifugation $(3000 \times \mathrm{g}, 10 \mathrm{~min})$ were kept at $-80{ }^{\circ} \mathrm{C}$ for further analysis. sT was 
measured in the laboratory of Teikyo University (Tokyo, Japan). For men aged 20-40 years, median sT is $139.4 \mathrm{pg} / \mathrm{ml}$ (10th

to 90 th percentile, $43.8-288.0 \mathrm{pg} / \mathrm{ml}$ ). The intra- and inter-assay coefficients of variance were $3.9 \%-8.8 \%$ and $6.7 \%-8.0 \%$, respectively. Slight cross-reactions with other natural steroids in the human body and their profiles were as follows: dihydrotestosterone, $2.5 \%$; androstenedione, $0.85 \%$; others, $<0.1 \% .^{17}$

\section{Depressive symptoms}

Depressive symptoms were considered as secondary exposures in light of their usefulness in predicting fall risk according to the literature and their potentially close association with testosterone deficiency. ${ }^{4}{ }^{12}$ Depressive symptoms were assessed using the 10-item version of the Center for Epidemiological Studies Depression Screening Index (CES-D). The cutoff score for depressive symptoms was set as a score of 10 or greater, as was recommended in the literature. ${ }^{18}$ While data on physician-diagnosed depression was unavailable in the LOHAS study, we assumed its use would considerably underestimate the true proportion of depression, as previous studies have shown that depression is underdiagnosed more often by Japanese physicians than those in other countries due to stigma related to psychiatric disorders within Japanese society. ${ }^{19,20}$

\section{Clinical outcomes}

We examined "any fall over a one-month period" as the clinical outcome based on subjects' answers to the question, "Over the past year, have you fallen down?" to establish fall history. Subjects who responded, "Yes", were then asked the follow-up question, "How many times have you fallen down over the past month?" with responses of "zero", "once", "twice", or "three or more times" allowed. Those who reported at least "once" were considered to have had "any fall" over the previous month. Regarding our decision to examine falls over the previous month rather than the previous year, we felt that reverse-causality would be more unavoidable if we were to use fall over the past year rather than the past month in investigating relationship with present sT level, and a systematic review has suggested that recalling fall experience at a one-year interval might underestimate true fall incidence compared to recalling incidence at a one-month interval. ${ }^{21}$

\section{Measurement of potential confounding variables}

Potential confounding variables examined in the present study were sociodemographic characteristics including age, exercise habit, and living alone, as well as the presence of cerebrovascular disease and the presence of incontinence, all obtained via self-reported questionnaire; body mass index and blood pressure, as measured by local nurse practitioners; hypertension, defined as systolic blood pressure $\geq 140 \mathrm{mmHg}$ and diastolic blood pressure $\geq 90 \mathrm{mmHg}$ or by individuals reporting attending a physician for treatment; diabetes, defined as having glycosylated hemoglobin values $\geq 6.1 \%$, as described by the Japanese Diabetes Society (equivalent to $\geq 6.5 \%$ described in National Glycohemoglobin Standardization Program [NGSP] values $^{22}$ ) or by individuals reporting attending a physician for treatment; and Timed Up and Go (TUG) test. Individuals were 
considered to engage in exercise if they answered "Yes" to the question concerning whether or not they had participated in moderate physical activity (making the individual breathe somewhat harder than normal and including situations such as carrying light loads, bicycling at a regular pace, or doubles tennis) in the previous seven days. Individuals were considered to have incontinence if they answered "once a week or more" to the question of whether or not they leaked urine because they could not defer the sudden urge to urinate. The TUG test, in which individuals are timed when rising from a chair, walking 3 $\mathrm{m}$, and turning to return to sit on the chair, is considered to be a reflection of function in gait, balance, and mobility ${ }^{23}$ with a greater score indicating more mobility problems.

\section{Statistical analysis}

Participants with complete data were entered into primary analyses. Statistical analyses were conducted using Stata version 11.0 (Stata Corp., College Station, TX, USA). sT, depressive symptoms, sociodemographic characteristics, comorbidities, TUG, and any fall over the previous month were described. Box plots for sT stratified by age categories were also created. Effect measures in the present study were odds ratios (ORs) of sT and depressive symptoms for likelihood of having any fall in the past month estimated using logistic regression models. To estimate adjusted ORs, the potential confounding variables described above were simultaneously forced into the models along with sT and depressive symptoms.

Given that the association of sT (as continuous variables) and falls might have been non-linear, as such non-linear relationships are well-established for several hormonal systems in the endocrinology literature, separate models were constructed to assess the shape of the association between sT and falls, where sT was included as (1) a linear variable, (2) a log-transformed variable, (3) a transformation using restricted cubic splines with 3 knots, and (4) 5 quintiles. To assess the fitness of these models, the Akaike Information Criterion (AIC), which is a likelihood-based measure that adds a penalty for model complexity, were reported. ${ }^{24}$

While nonlinear models were superior to the linear model in terms of the AIC, the 5-quintile model and the restricted cubic spline model were similar to one another (Table S1). We therefore chose the restricted cubic spline model for primary analysis, as this model provided a good fit and was the most parsimonious, and we based all further testing on it. In this model, the 90th percentile of sT was chosen as a reference, as it corresponds to the median of the highest quintile. The potential effect modification of depressive symptoms on the association between sT and any fall over the previous month was examined by likelihood ratio test, adding interaction pair (the product terms of sT with depressive symptoms) to the logistic regression models.

\section{Sensitivity analysis}

In addition to the above, we also conducted two sensitivity analyses. First, the association between sT and fall was reported, with 5 quintiles of sT included, citing the top category of the quintiles as reference. Second, the association between sT and 
falls was examined including 280 participants with missing covariate values. In this analysis, restricted cubic splines with 3 knots were used with adjustment for covariates via the missing indicator method. Third, CES-D was used as exposure instead of depressive symptoms, and the association between CES-D score and fall was examined. $\mathrm{P}<0.05$ was considered statistically significant.

\section{$\underline{\text { Results }}$}

Of the 869 men who underwent the health check exam (Figure 1), 58 and 13 participants were missing data for sT and any falls, respectively. After exclusion of 36 participants with poor-quality saliva specimens (due to inadequate amount obtained or suspected blood contamination), $762(87.7 \%)$ remained with both sT and outcome variables. After exclusion of a further 280 participants with at least one confounding variable missing, the remaining 482 participants were ultimately entered into the primary analyses.

Baseline characteristics are presented in Table 1. Median age in the present study was 70 years (10th to 90th percentile, 63-78 years). The prevalence of hypertension and diabetes were $69.3 \%$ and $14.5 \%$, respectively. Median sT was $58.7 \mathrm{pg} / \mathrm{ml}$ (10th to 90 th percentile, $26.3-146 \mathrm{pg} / \mathrm{ml}$ ). Box plots for sT showed that median sT was highest among those aged 60-64 years and lowest among those aged over 80 years (Figure 2). The prevalence of depressive symptoms was $11.6 \%$. The prevalence of any fall over the previous month was $10.8 \%$.

Sociodemographic characteristics were similar between participants with and without missing confounding variables (Table S2) except for exercise habit. As a group, their sT, TUG value, proportion with depressive symptoms, and proportions of any fall over the one-month period examined were similar. As a group, baseline characteristics among participants in the primary analysis set were similar to those among whole older men, except for with respect to exercise habit, age (slightly younger), and TUG value (slightly lower)(Table S3).

In the covariate-adjusted restricted cubic spline model, the splines demonstrated a nonlinear relationship between sT and falls $(\mathrm{P}=0.0021$ for non-linearity). The estimated shape of the sT is shown in Figure 3, suggesting that the lower the sT level, the greater the likelihood of having any fall, citing 90 percentile of sT as a reference. In this model, the adjusted ORs of depressive symptoms for any fall were 3.49 (95\% confidence interval [CI] 1.52-8.04). We noted no apparent effect modification by depressive symptoms on the association between the sT and any fall ( $\mathrm{P}=0.079$ for interaction).

To estimate the strength of the association of combination of sT levels and depressive symptoms, estimated odds ratios of combination of a given sT and depressive symptoms were shown in Table 2. Compared with 90 percentile of sT, lower sT is associated with greater likelihood of having any fall. For example, OR for any fall in 5 percentile of sT is 4.23 fold (95\% CI 1.66 - 10.8). Compared with combination of 90 percentile of sT and no depressive symptoms, OR for any fall in combination of 5 percentile sT and depressive symptoms is 14.8 fold (95\% CI $3.76-58.0)$.

In sensitivity analysis using sT quintiles citing the top quintile as reference, the lowest quintile of sT is associated 
with greater likelihood of having any fall (Table S4). Similarly, depressive symptoms were associated with an increased likelihood of having any fall, although this quintile model performed only slightly worse than the spline model (Table S1). Another sensitivity analysis using a restricted cubic spline model among 762 participants showed similar associations between sT and increased ORs for any fall (Figure S1). When CES-D was used as exposure instead of depressive symptoms, CES-D score was associated with any fall (Table S5).

\section{$\underline{\text { Discussion }}$}

In this cross-sectional study of community-dwelling older men, low testosterone levels were independently associated with any falls over the previous month. We also observed an increased association between depressive symptoms with any fall. In addition, combination of low testosterone levels and depressive symptoms had stronger association with falls than either parameter alone. These findings suggest that clinicians should be aware of the increased risk of falls in older men with testosterone deficiency or depressive symptoms.

Our findings regarding the relationship between low testosterone levels and increased likelihood of falling concur with results from previous studies. A study in France among adults aged 50-85 years found that low free testosterone levels were associated with increased likelihood of suffering falls over the last year (OR of decreased level of free testosterone vs. normal level: 1.54). ${ }^{11}$ Similarly, reduced bioavailable testosterone levels were found to be associated with increased likelihood of suffering falls in the future among adults in the US aged 65-99 years (risk ratio of the lowest quartile of bioavailable testosterone vs. the highest quartile: 1.40). ${ }^{10}$ Other studies also noted an association between low total testosterone levels and increased likelihood of suffering falls in the future among adults in the US aged over 65 years (OR of the highest quartile of total testosterone vs. the lowest quartile: 0.22$)^{9}{ }^{9}$

However, conflicting results have also been reported. For example, a study in the Netherlands noted no association between total testosterone levels and incident falls among adults aged $65-88$ years. ${ }^{13}$ Such conflicting findings may be explained in part by differences between measured fractions of circulating testosterone and ones in commercial assays, as well as by inclusion of depressive symptoms and other confounding factors for adjustment. As such, increased likelihood of suffering a fall among individuals with low testosterone levels may due to their increased likelihood of having depressive symptoms in studies where such symptoms are not assessed. Only in the Netherlands study evaluated the relationship between testosterone level and falls, with statistical adjustment for presence of depressive symptoms. In addition to these previous findings, our present findings here further noted that joint effect of low testosterone levels and depressive symptoms on falls in combination compared with the joint reference category (90 percentile of sT and no depressive symptoms) had multiplicative effect on falls (i.e. each exposures was independently associated with falls). In addition, using splines, we noted a continued increase in risk of falling among subjects with relatively low testosterone levels; by definition, quartiles or quintiles cannot detect an increase in risk below the $25^{\text {th }}$ centile or $20^{\text {th }}$ centile of exposure, respectively. ${ }^{25}$ 
We feel that our findings here will influence the activities of physicians and health-policymakers for several reasons.

First, both low testosterone levels and depressive symptoms are potentially modifiable risk factors for falls. Testosterone deficiency can be managed with testosterone replacement therapy, and depressive symptoms can be managed with a combination of cognitive behavioral therapy and antidepressants; individuals with both testosterone deficiency and depressive symptoms may be able to manage their condition with a combination of these therapies. Physicians who encounter individuals with suspected testosterone deficiency should therefore carefully assess the presence of depressive symptoms to reduce further risk of falls. An international survey conducted among physicians treating testosterone deficiency showed that depressive symptoms are considered to be one of main symptoms, other than sexual problems, related to testosterone deficiency - a finding which supports the potential coexistence of depressive symptoms with testosterone deficiency. ${ }^{14}$ Second, screening for low testosterone levels in the saliva using ELISA may be a reasonable health plan for community-dwelling older men to stratify the fall risk, as ELISAs are more cost-efficient ( $\$ 5$ per sample) and easier to perform than radioimmunoassay and liquid chromatography/mass spectrometry. ${ }^{26}$ Third, any biological connection between low testosterone levels and falls may be independent of mobility function, as we estimated the relationship between testosterone and falls with adjustment for TUG, which reflects gait, balance, and mobility. For example, reduced cognitive function may be involved as a non-mobility-related intermediate pathway, as low testosterone levels may be associated with visuospatial ability or vigilance. ${ }^{27,28}$

Several strengths to the present study warrant mention. First, we demonstrated the relationship between testosterone and falls among community-dwelling older adults, adjusting for confounding variables such as depressive symptoms and TUG, which are potentially related to both testosterone and falls. Second, we showed that the strength of the association between combination of low testosterone level and depressive symptoms and falls might be larger than that of low testosterone or depressive symptoms alone; specifically, the strength of the association appears to be equal to the product of the two exposures in terms of OR. These findings provide a basis for thoughtful consideration of how to reduce risk of falls in older men with both testosterone deficiency and depressive symptoms.

However, several limitations to the present study also warrant mention. First, although sT is a reliable and suitable metric for evaluating testosterone levels in population-based studies, ${ }^{29-31}$ the clinical guideline for the diagnosis of hypogonadism does not recommend its clinical use, as the methodology has not been standardized and adult male ranges are not yet available in most hospital or reference laboratories. ${ }^{32}$ Therefore, in actual clinical practice, serum testosterone levels should be measured to diagnose hypogonadism. Further prospective studies are warranted to determine whether or not late-onset hypogonadism diagnosed via serum test and depressive symptoms are jointly associated with falls. The prevalence of late-onset hypogonadism is also known to rise with increasing body mass index (BMI) and number of coexisting illnesses. Median BMI in our population was much lower than mean values reported in western countries. ${ }^{9-11,13}$ However, a study among Korean men aged 21-79 years showed that the subjects' mean BMI was 24.5, and that even in the lowest quintile by 
serum testosterone level (113-378 ng/dL), BMI was 25.2. ${ }^{33}$ Given these previous findings, hypogonadism among Asian people diagnosed by blood testing may be found among individuals with relatively low BMI more often than among Caucasians with BMI in the same range. Further, some endocrine specialists question the validity of sT measured by ELISA. However, previous studies have shown that sT levels obtained via ELISA share good correlation with those obtained via liquid chromatography/mass spectrometry, ${ }^{26}$ and sT has also been shown to be closely correlated to serum free testosterone. ${ }^{34}$ However, in addition to differences noted based on assay adopted, blood contamination in saliva might also influence testosterone concentration. ${ }^{35}$ As such, in the present study, saliva with suspected blood contamination was excluded from analyses. Even taken together with these drawbacks, however, utilization of sT still facilitates easily conducted, minimally invasive screening methods for evaluating testosterone levels on a population basis. Second, non-prescriptional use of testosterone or methyl-testosterone could not be recorded. However, use of such medication in our population is unlikely, as these compounds require a physician's prescriptions and are not available as over-the-counter drugs in Japan. Third, the cross-sectional design of the present study means that we cannot attribute causality from the associations between testosterone and depressive symptoms and falls. While we believe that low testosterone being a cause of falling is biologically plausible, association between low testosterone or depressive symptoms and falls might be explained by reverse causality. Fourth, we were unable to include other medication usage and all chronic conditions in our analyses, and given that use of antihypertensives, sedatives, or antidepressants are also potential risk factors for falls, ${ }^{4,36}$ the association between salivary testosterone and falls in the present study might be confounded by these factors. To mitigate this limitation, we adjusted for individuals with hypertensive problems. Effect of depressive symptoms on falls might be mediated partly by sedatives or antidepressants, as individuals with depressive symptoms are often prescribed these agents. The literature suggests that use of serotonin selective reuptake inhibitor is indeed associated with increased salivary testosterone levels, ${ }^{37}$ and that serotonin selective reuptake inhibitor is associated with falls. ${ }^{36}$ However, given that we adjusted for depression symptoms, we therefore believe it unlikely that any association between sT and falls was confounded by antidepressants. While arthritis (including osteoarthritis) are prevalent in elderly and also potential risk factors for falls, ${ }^{38}$ its burden on functional mobility should be reflected by TUG. As such, we believe that these confounding factors had negligible effects on our analyses. Fifth, a high rate of missing data was noted in the primary analyses. In sensitivity analyses using $87.7 \%$ of the older men in the health check-up exam showed an association similar to, but slightly smaller than, that noted in primary analyses between low sT and falls (Figure S1). We therefore cannot completely exclude the possibility that our findings in the primary analysis may suffer from selection bias.

\section{$\underline{\text { Conclusion }}$}

In conclusion, both lower testosterone and depressive symptoms were found to be separately associated with any falls over a one-month period among community-dwelling older men. Further, having both low testosterone levels and 
depressive symptoms might imbue a greater likelihood of having falls than either metric alone. Causality of these associations should be confirmed in future prospective studies with adjustment for potential confounding by more numbers of chronic illnesses.

\section{$\underline{\text { Acknowledgement }}$}

The authors have nothing to disclose.

\section{$\underline{\text { References }}$}

1. Tinetti ME, Speechley M, Ginter SF. Risk factors for falls among elderly persons living in the community. $N$ Engl $J$ Med. Dec 29 1988;319(26):1701-1707.

2. Rubenstein LZ, Josephson KR. The epidemiology of falls and syncope. Clin. Geriatr. Med. May 2002;18(2):141-158.

3. Moreland JD, Richardson JA, Goldsmith CH, Clase CM. Muscle Weakness and Falls in Older Adults: A Systematic Review and Meta-Analysis. J. Am. Geriatr. Soc. 2004;52(7):1121-1129.

4. Tromp AM, Smit JH, Deeg DJH, Bouter LM, Lips P. Predictors for Falls and Fractures in the Longitudinal Aging Study Amsterdam. Journal of Bone and Mineral Research. 1998;13(12):1932-1939.

5. Kurita N, Yamazaki S, Fukumori N, et al. Overactive bladder symptom severity is associated with falls in community-dwelling adults: LOHAS study. BMJ open. 2013.

6. Allan CA, McLachlan RI. Age-related changes in testosterone and the role of replacement therapy in older men. Clinical Endocrinology. 2004;60(6):653-670.

7. Krasnoff JB, Basaria S, Pencina MJ, et al. Free Testosterone Levels Are Associated with Mobility Limitation and Physical Performance in Community-Dwelling Men: The Framingham Offspring Study. J. Clin. Endocrinol. Metab. June 1, 2010 2010;95(6):2790-2799.

8. Roy TA, Blackman MR, Harman SM, Tobin JD, Schrager M, Metter EJ. Interrelationships of serum testosterone and free testosterone index with FFM and strength in aging men. American Journal of Physiology - Endocrinology And Metabolism. August 1, 2002 2002;283(2):E284-E294.

9. Bischoff-Ferrari HA, Orav EJ, Dawson-Hughes B. Additive benefit of higher testosterone levels and vitamin D plus calcium supplementation in regard to fall risk reduction among older men and women. Osteoporosis Int. Sep 2008;19(9):1307-1314.

10. Orwoll E LLCMLM, et al. ENdogenous testosterone levels, physical performance, and fall risk in older men. Archives of Internal Medicine. 2006;166(19):2124-2131.

11. Szulc P, Claustrat B, Marchand F, Delmas PD. Increased risk of falls and increased bone resorption in elderly men 
with partial androgen deficiency: the MINOS study. J Clin Endocrinol Metab. Nov 2003;88(11):5240-5247.

12. Joshi D, Van Schoor NM, De Ronde W, et al. Low free testosterone levels are associated with prevalence and incidence of depressive symptoms in older men. Clinical Endocrinology. 2010;72(2):232-240.

13. Schaap LA, Pluijm SM, Smit JH, et al. The association of sex hormone levels with poor mobility, low muscle strength and incidence of falls among older men and women. Clin Endocrinol (Oxf). Aug 2005;63(2):152-160.

14. Gooren LJ, Behre HM, Saad F, Frank A, Schwerdt S. Diagnosing and treating testosterone deficiency in different parts of the world. Results from global market research. The Aging Male. 2007;10(4):173-181.

15. Gruenewald DA, Matsumoto AM. Testosterone supplementation therapy for older men: Potential benefits and risks. J. Am. Geriatr. Soc. Jan 2003;51(1):101-115.

16. Otani K, Takegami M, Fukumori N, et al. Locomotor dysfunction and risk of cardiovascular disease, quality of life, and medical costs: design of the Locomotive Syndrome and Health Outcome in Aizu Cohort Study (LOHAS) and baseline characteristics of the study population. J Orthop Sci. May 2012;17(3):261-271.

17.

IBL

INTERNATIONAL

$\mathrm{GmbH}$.

Testosteron

Saliva

ELISA.

http://www.ibl-international.com/media/catalog/product/R/E/RE52631_IFU_en_Testosteron_Saliva_ELISA_2013-0

4_sym3.pdf. Accessed Accessed Sep 17, 2013.

18. Andresen EM, Malmgren JA, Carter WB, Patrick DL. SCREENING FOR DEPRESSION IN WELL OLDER ADULTS - EVALUATION OF A SHORT-FORM OF THE CES-D. Am. J. Prev. Med. Mar-Apr 1994;10(2):77-84.

19. MINO Y, AOYAMA H, FROOM J. Depressive Disorders in Japanese Primary Care Patients. Family Practice. December 1, 1994 1994;11(4):363-367.

20. Lopes AA, Albert JM, Young EW, et al. Screening for depression in hemodialysis patients: Associations with diagnosis, treatment, and outcomes in the DOPPS. Kidney Int. 2004;66(5):2047-2053.

21. Ganz DA, Higashi T, Rubenstein LZ. Monitoring Falls in Cohort Studies of Community-Dwelling Older People: Effect of the Recall Interval. J. Am. Geriatr. Soc. 2005;53(12):2190-2194.

22. Seino Y, Nanjo K, Tajima N, et al. Report of the Committee on the Classification and Diagnostic Criteria of Diabetes Mellitus. Journal of Diabetes Investigation. 2010;1(5):212-228.

23. van Iersel MB, Munneke M, Esselink RAJ, Benraad CEM, Olde Rikkert MGM. Gait velocity and the Timed-Up-and-Go test were sensitive to changes in mobility in frail elderly patients. Journal of Clinical Epidemiology. 2008;61(2):186-191.

24. Akaike H. A new look at the statistical model identification. Automatic Control, IEEE Transactions on. 1974;19(6):716-723.

25. Bennette C, Vickers A. Against quantiles: categorization of continuous variables in epidemiologic research, and its discontents. BMC Med Res Methodol. 2012;12:21. 
26. Yasuda M, Honma S, Furuya K, et al. Diagnostic significance of salivary testosterone measurement revisited: using liquid chromatography/mass spectrometry and enzyme-linked immunosorbent assay. Journal of Mens Health. Mar 2008;5(1):56-65.

27. Salminen EK, Portin RI, Koskinen A, Helenius H, Nurmi M. Associations between Serum Testosterone Fall and Cognitive Function in Prostate Cancer Patients. Clinical Cancer Research. November 15, 2004 2004;10(22):7575-7582.

28. Thilers PP, MacDonald SWS, Herlitz A. The association between endogenous free testosterone and cognitive performance: A population-based study in 35 to 90 year-oldmen and women. Psychoneuroendocrinology. 2006;31(5):565-576.

29. Arregger AL, Contreras LN, Tumilasci OR, Aquilano DR, Cardoso EML. Salivary testosterone: a reliable approach to the diagnosis of male hypogonadism. Clinical Endocrinology. 2007;67(5):656-662.

30. Gavrilova N, Lindau ST. Salivary Sex Hormone Measurement in a National, Population-Based Study of Older Adults. The Journals of Gerontology Series B: Psychological Sciences and Social Sciences. November 1, 2009 2009;64B(suppl 1):i94-i105.

31. Wang C, Plymate S, Nieschlag E, Paulsen CA. SALIVARY TESTOSTERONE IN MEN - FURTHER EVIDENCE OF A DIRECT CORRELATION WITH FREE SERUM TESTOSTERONE. Journal of Clinical Endocrinology \& Metabolism. 1981;53(5):1021-1024.

32. Wang $\mathrm{C}$, Nieschlag E, Swerdloff R, et al. Investigation, treatment and monitoring of late-onset hypogonadism in males: ISA, ISSAM, EAU, EAA and ASA recommendations. European Journal of Endocrinology. November 1, 2008 2008;159(5):507-514.

33. Hong D, Kim Y-S, Son ES, et al. Total testosterone and sex hormone-binding globulin are associated with metabolic syndrome independent of age and body mass index in Korean men. Maturitas. 2013;74(2):148-153.

34. Gröschl M. Current Status of Salivary Hormone Analysis. Clinical Chemistry. November 1, 2008 2008;54(11):1759-1769.

35. Granger DA, Shirtcliff EA, Booth A, Kivlighan KT, Schwartz EB. The "trouble" with salivary testosterone. Psychoneuroendocrinology. 2004;29(10):1229-1240.

36. Thapa PB, Gideon P, Cost TW, Milam AB, Ray WA. Antidepressants and the risk of falls among nursing home residents. N. Engl. J. Med. Sep 1998;339(13):875-882.

37. Giltay EJ, Enter D, Zitman FG, et al. Salivary testosterone: Associations with depression, anxiety disorders, and antidepressant use in a large cohort study. Journal of Psychosomatic Research. 2012;72(3):205-213.

38. Arden NK, Nevitt MC, Lane NE, et al. Osteoarthritis and risk of falls, rates of bone loss, and osteoporotic fractures. Arthritis \& Rheumatism. 1999;42(7):1378-1385. 


\section{Table legend:}

Table 1. Baseline characteristics of the analysis population

Table 2. Adjusted odds ratio of any fall over the previous month in men with aged over 60 years ${ }^{\mathrm{a}}$

${ }^{a}$ Logistic regression analysis with restricted cubic spline adjusted for covariates (age, body mass index, exercise habit, living alone, timed up and go, depressive symptoms, incontinence, hypertension, diabetes, cerebrovascular disease). Combination of 90 percentile of the salivary testosterone level (i.e. median of the fifth quintile) and not having depressive symptoms was cited as reference.

\section{Figure legend:}

Figure 1. Flow chart of study participants

sT: salivary testosterone. Sum of number of participants with missing values for each covariate exceeds 280 because 90 participants had missing values for 2 or more covariates.

\section{Figure 2. Box plots for salivary testosterone levels stratified by age categories}

White line in the boxes indicates median of sT in each category. Top and bottom of the boxes indicate 75 th and 25th percentiles of sT in each category, respectively.

\section{Figure 3. Adjusted odds ratio of any fall over the previous month in men with aged over 60 years ${ }^{\mathrm{a}}$}

${ }^{a}$ Logistic regression analysis with restricted cubic spline adjusted for covariates (age, body mass index, exercise habit, living alone, timed up and go, depressive symptoms, incontinence, hypertension, diabetes, cerebrovascular disease). Reference was set as 90th percentile of the salivary testosterone level (i.e. median of the fifth quintile). The left vertical axis shows OR in log scale. Solid line indicates point estimates of OR. Dotted lines indicate CI. Gray bars indicate frequency of the salivary testosterone values. The right vertical axis shows frequency of each gray bar. OR: odds ratio, CI: confidence interval 
Table 1. Baseline characteristics of the analysis population

\begin{tabular}{lcc}
\hline Total $(\mathrm{N}=482)$ & No falls over the & Any fall over the \\
& previous month $(\mathrm{N}=435)$ & previous month $(\mathrm{N}=47)$ \\
\hline
\end{tabular}

Age, $\mathbf{y}$

Median

10th to 90th percentile

Body mass index, $\mathrm{kg} / \mathrm{m}^{2}$

Median

10th to 90th percentile

Exercise habit, n (\%)

Living alone, $\mathrm{n}(\%)$

Incontinence, $\mathrm{n}(\%)$

Hypertension, n (\%)

Cerebrovascular disease, $n(\%)$
70

63-78

63-78

63-79

$264(54.8)$

$61(12.7)$

$38(7.9)$

$334(69.3)$

$28(5.8)$
24.4

23.8

20.2-27.4

21.1-26.6

$235(54.0)$

$29(61.7)$

$53(12.2)$

$8(17.0)$

$35(8.1)$

$3(6.4)$

$302(69.4)$

$32(68.1)$

$21(4.8)$ 
Diabetes, n (\%)

Timed Up and Go, s

Median

10th to 90th percentile

Depressive symptoms, n (\%)

Salivary testosterone, pg/ml

Median

26.3-146

$70(14.5)$

7.1

$5.4-9.5$

$56(11.6)$

10th to 90th percentile
$64(14.5)$

7 (14.9)

7.1

8.0

$5.3-9.4$

5.7-10.5

45 (10.3)

$11(23.4)$
61.0

47.8

25.8-133 
Table 2. Adjusted odds ratio of any fall over the previous month in men aged over 60 years ${ }^{\mathrm{a}}$

Adjusted odds ratio (95\% confidence interval) for

Salivary Testosterone

any fall over the previous month

\begin{tabular}{cccc}
\hline & & Participants without & Participants with \\
percentile & $p g / m l$ & Depressive symptoms & Depressive symptoms \\
\hline 5 & 21.8 & $4.23(1.66-10.8)$ & $14.8(3.76-58.0)$ \\
10 & 26.3 & $3.74(1.58-8.83)$ & $13.1(3.54-48.2)$ \\
50 & 43.1 & $2.39(1.34-4.26)$ & $8.35(2.78-25.1)$ \\
70 & 58.9 & $1.67(1.17-2.38)$ & $5.83(2.23-15.3)$ \\
90 & 80.3 & $1.21(1.03-1.43)$ & $4.23(1.77-10.1)$ \\
95 & 146.5 & 1 reference] & $3.49(1.52-8.04)$ \\
\hline
\end{tabular}




\section{Figure 1}

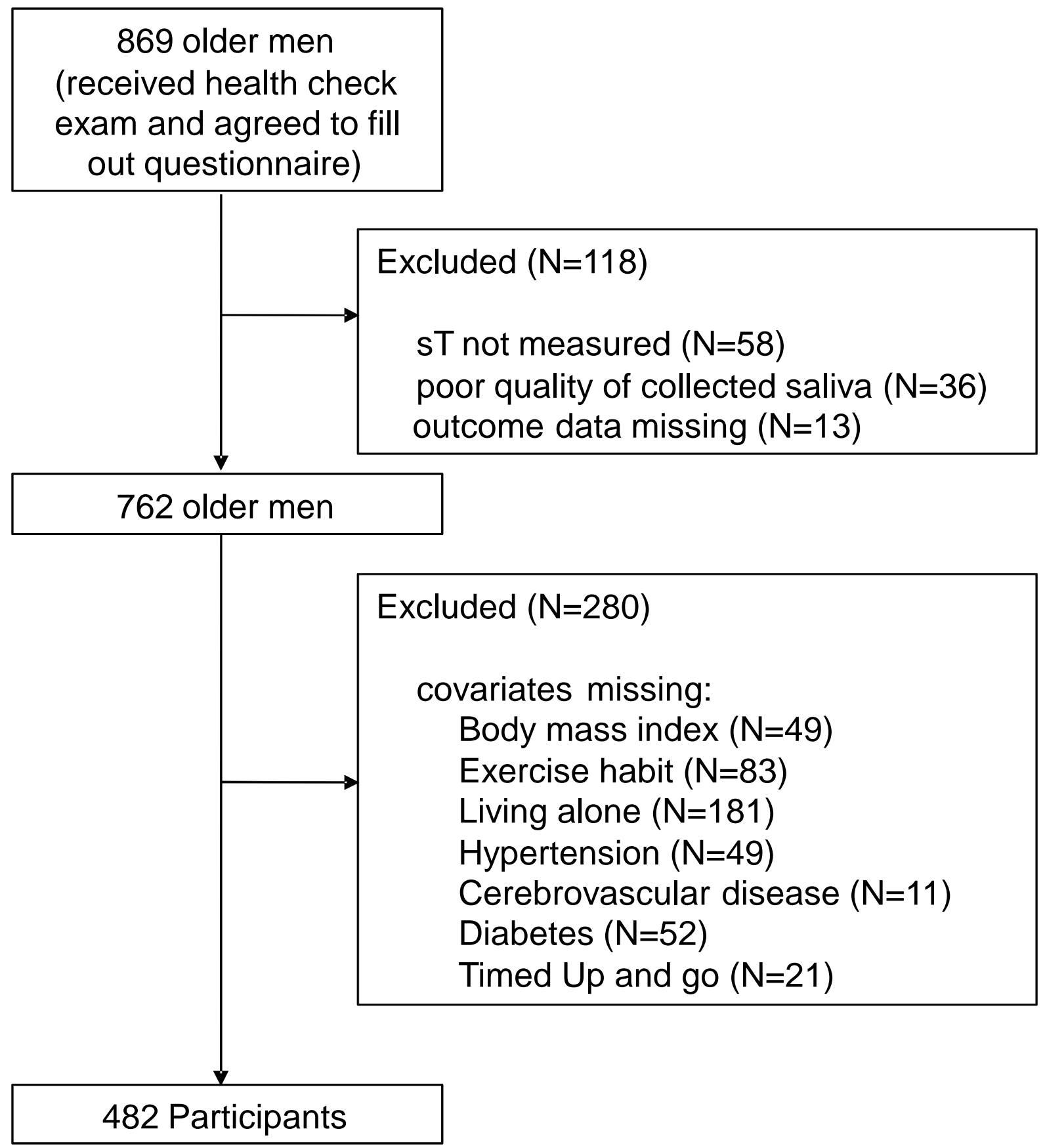




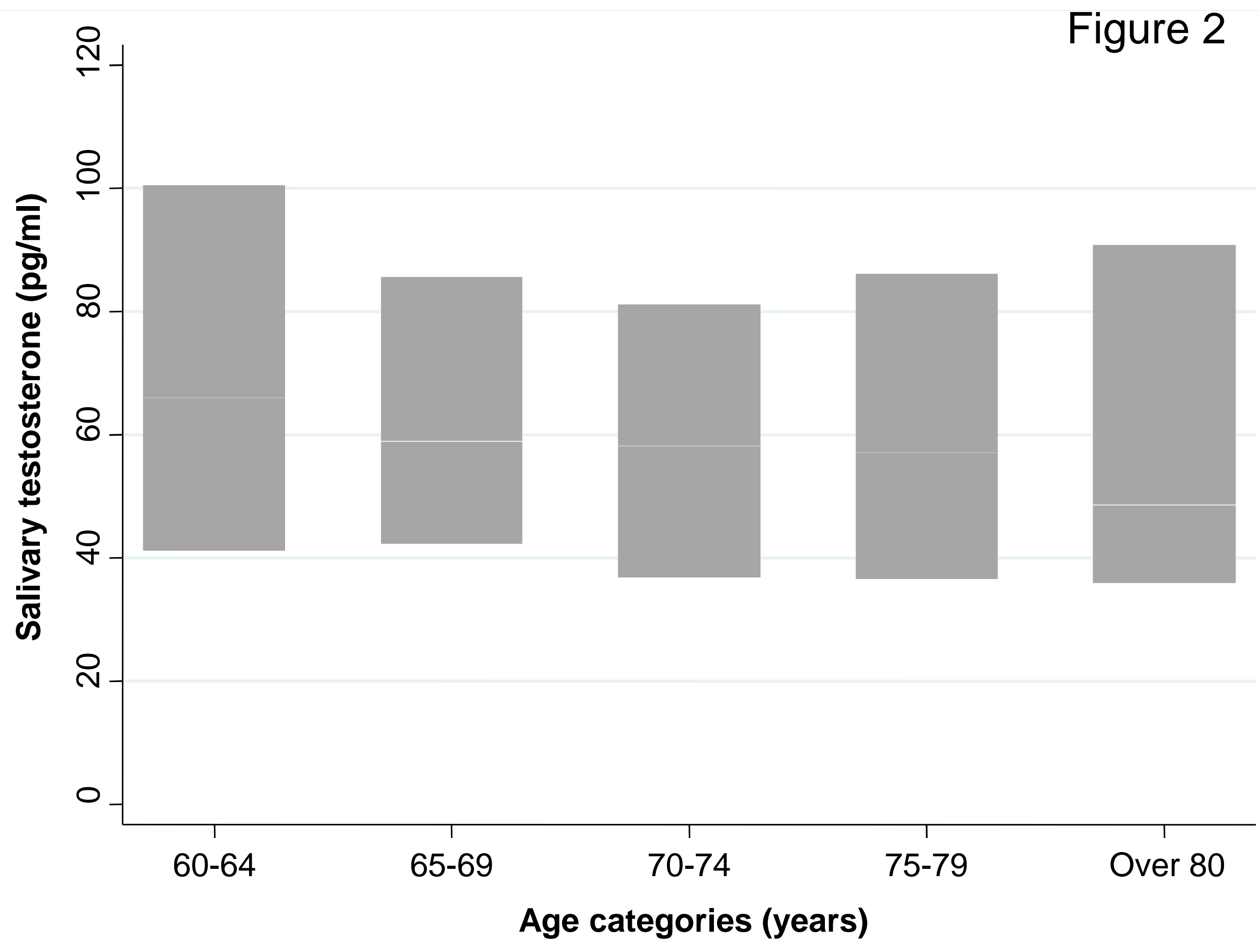


Figure 3

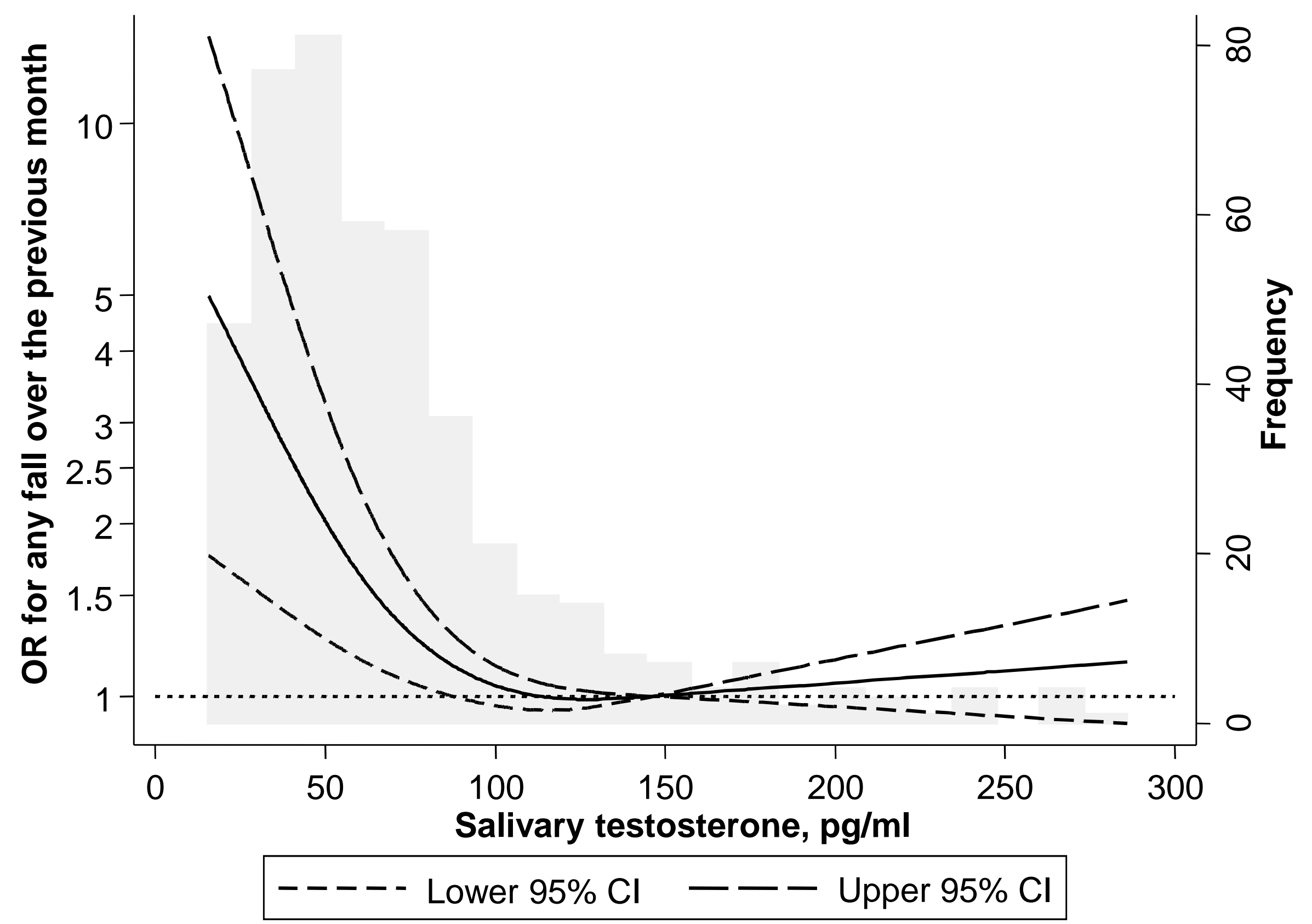

